\title{
神经节苷脂对酪胺的保护作用 及与酪胺的结合*
}

高平张宗俩

(北京大学生物系)

Woolley 及 Gommi 报道 ${ }^{[1]}$, 用神经氨酸酶处理的真菌制备对 5-差色胺的敏感性消失后, 可以用加人神经节苷脂,特别是 $\mathrm{GD}_{3}$, 使其恢复. 核磁共振的研究也指出, 在 5-羟色胺与神 经节苩脂之间有强烈交互作用; 在 $60 \mathrm{MH}_{2}$ 处胺的芳族共振有显著地加宽 ${ }^{[2]}$, 所以,很多人认 为,神经节苷脂可能是 5-羟色胺的受体. McIlwain 报道 ${ }^{[3]}$, 用鱼精蛋白处理脑切片后, 神经组 织的兴奋性被破坏,也可以用加神经节苷脂而使其恢复。他建议,神经节苷脂与鱼精蛋白可能 结合, 形成一个复合物。

在昆虫中, 酪胺曾被鉴定为 DDT 中毒蚌蠊的血淋巴内的神经毒素 ${ }^{[4]}$. 它在低浓度时引 起兴奋,而在高浓度时, 造成神经传导的阻断.

初步试验发现,神经节苷脂能保护美洲蚌䎓, 不受酪胺对神经的破坏作用。本文研究了神 经节苷脂对酪胺的保护作用及其机制, 探讨是否神经节苷脂能与酪胺结合, 形成复合物.

\section{一、材料与方法}

材料 [ $\left.{ }^{3} \mathrm{H}\right]$-酪胺 $(1.17 \mathrm{mCi} / \mathrm{mg})$ 由中国科学院原子能研究所供给.硅胶 60-薄膜层析 板 $\left(0.25 \mathrm{~mm}\right.$ 厚)由 Merck 购得. 猪脑神经节葉脂按照 Ledeen 的方法 ${ }^{[s]}$ 提取.

结合反应将猪脑神经节葉脂及 $\left[{ }^{3} \mathrm{H}\right]$-酪胺分别溶于氯仿甲醇中 $(1: 1, V / V)$, 以后以 $1: 3(W / W)$ 的比例混合. 在 $4^{\circ} \mathrm{C}$ 中保温 $24 \mathrm{~h}$, 使反应达到平衡.

单相薄层层析(用 TLC 板, $5 \times 20 \mathrm{~cm}$ ), 神经节苷脂及 $\left[{ }^{3} \mathrm{H}\right]$-酪胺的混合物以 $1.5 \mathrm{~cm}$ 的线 状点样 $\left(0.4 \mathrm{~cm}\right.$ 长). 单相薄层层析连续用三个层析系统: (1) 氯仿, $1 \mathrm{~h}, 20^{\circ} \mathrm{C},(2)$ 氯仿: 甲醇: 水 $(70: 30: 4), 2 \mathrm{~h} ;(3)$ 氯仿: 甲醇: $0.25 \%$ 氯化钾 $(60: 35: 8), 5 \mathrm{~h}$. 放射自显影是用 $\mathrm{X}$ 光胶片覆 盖在层析板上, 黑暗处放置 2 个月. 取出后, 用 Svennerholm ${ }^{[6]}$ 的间苯二酚法显色（间苯二酚$\mathrm{HCl}$ 试剂的配制法如下: $\mathrm{mol} / \mathrm{l}$ 硫酸酮 $0.025 \mathrm{ml}$, 浓盐酸 $8 \mathrm{ml}, 2 \%$ 间苯二酚 $1 \mathrm{ml}$, 加水 $1 \mathrm{ml}$, 混匀,在使用前 $4 \mathrm{~h}$ 配制)。喷客间苯二酚试剂后, 盖以玻片, 以防盐酸挥发，用夹子夹住，在 $95^{\circ} \mathrm{C}$ 烘烤 $20 \mathrm{~min}$, 含唾液酸物质会产生特征的紫色. 自显影的色斑可与层析的色斑进行比较 鉴定.

对于双相薄层层析(用 TLC 板 $10 \times 10 \mathrm{~cm}$ )，第一相的层析系统为氯仿：甲醇： $0.2 \%$ 氯化钻水溶液 $(50: 40: 10)$, $3 \mathrm{~h}$ ，第二相为氯仿：甲酧： $2.5 \mathrm{~mol} / 1$ 铵 $(50: 40: 10)$, $3 \mathrm{~h}$, 也用间苯 二酚 $\mathrm{HCl}$ 试剂喷学显色. 在放射自显影试验中,将色用手术刀刮下，将硅胶粉置于内㷧瓶

本文 1986 年 7 月 26 日收到.

*国家目然科学基金资助项目. 
中, 加人 $10 \mathrm{ml}$ 闪䐆液 (甲苯含 $\mathrm{PPO}^{*} 0.4 \%, \mathrm{POPOP}^{*} 0.01 \%$ ), 摇匀,过夜使成均相, 在液体闪: 伢计数器 (Beckman LS-9800) 上计数。根据单个点的结合物计数在总的结合物计数中的百 分率来计算结合率.

电生理试验将雄性美洲蚌䖢成虫(最后一次蜕皮后 2-6 周)切除足和翅,腹面向上固 定于标本板上,除去部分表皮及一部分肌肉及其他组织, 暴笿腹部第六及第三神经节. 在神经 节下各垫一小块胶片，在第六腹神经节周围用凡土林防干油做一圆形楷，将一小块滤纸片夏 盖在神经节上,滴加生理盐水，用铜丝小钩电极做记录电极，钩起第三腹神经节，上復防干油以 延长引导的间. 参考电极埋于腹部节片下. 记录电极与 100 倍 AC 前极放大器相连, 放大器 的输出送往 SBR-1 型二线示波器和监听器.

为了灌流神经制备, 用了一个电子微量泵 (WLB-78-C 型), 保证了微量加样器不断地溥 流微量的液体. 将加样器的针头用一个操纵器固定在神经制备上面, 这样使得灌流的液体可 以十分准确地加在神经制备上,因而只要灌流的液体浓度不变,每次重复可以获得很大的一致 性。

为了使蚌蠊在解剖后安定下来,所有试验开始前,都先用生理盐水*灌流 $20 \mathrm{~min}$, 用吹耳球 对尾需吹气,以诱发神经冲动的产生做为神经反应的指标.

当昆虫标本达到正常, 则更换灌流液, 分别缓慢地将 $10^{-4} \mathrm{~mol} / 1$ 酪胺或 $10^{-4} \mathrm{~mol} / 1$ 酪胺 （含 $10^{-7} \mathrm{~mol} / 1$ 猪脑神经节苷脂）灌流于腹六神经节上. 用滤纸片将灌流废液引人培养血中, 神经脉冲信号由 Exercise-II 型 Z-80 单极计算机记录,并由日立-058 记录仪描出.

灌流的对照液即为酪胺溶于生理盐水中, 另将猪脑神经节苗脂按一定比例与酪胺混合, 使 终浓度中的酪胺与对炤液相同. 实验时的温度为 $23 \pm 2{ }^{\circ} \mathrm{C}$. 组间差异的显著性测定用 $t$ 检验 法(双尾)。

\section{二、结 果}

结合反应双相薄层层析后的液体闪㷧计数的结果显示出, $\left[{ }^{3} \mathrm{H}\right]-$ 酪胺与猪脑神经节 苷脂中的主要成分均能结合。其结合率分别为: GM1 $15.37 \pm 0.03 \%, G D 323.45 \pm 0.46 \%$, GD1a7.37 $\pm 0.03 \% ， G D 1 b 17.48 \pm 0.08 \%$ ，GT1b $22.82 \pm 0.50 \% ， G Q 1 b 14.05 \pm 0.11 \%$ 。

单向薄层层析的自显影结果显示出, 在 $\left[{ }^{3} \mathrm{H}\right]$ - 酪胺与神经节苷脂结合之后, 在 GD3 及 GT1b 处出现了两个新带 (图 1).

电生理试验对正常蚌蠊尾须吹气,有诱发反应产生. 当用 $10^{-4} \mathrm{~mol} / 1$ 酪胺灌流蚌蠊的: 第六腹神经节时, 出现的中毒征象如下: 自发性神经活动增加, 在 $21 \mathrm{~min}$ 时连串神经冲动发 生, 强度及频率均有增加, 神经冲动继续加强,持续 $3 \mathrm{~min}$ 后,在 $23 \mathrm{~min}$ 时完全阻断 (图 2). 这 可称为“阻断时间”,在这时候自发活动及诱发反应均全部消失.

灌流 $10^{-4} \mathrm{~mol} / 1$ 酪胺(其中含 $10^{-7} \mathrm{~mol} / 1$ 猪脑神经节苷脂)于蚌蠊第六腹神经节，神经冲动 的阻断时间可延迟至 $57 \mathrm{~min}$ (图 3). 更高浓度的神经节苷脂可能会防止其阻断. 因此,神经 节苷脂对于酪胺的破坏神经传导的作用有一定保护作用,至少可延迟其阻断的发生。

- PPO: 2,5-二苯基㗭唑; POPOP: 1,4-双 [5-苯基䍐㟇基-2] 苯; 蚌蝶生理盐水: $214 \mathrm{mmol} / 1 \mathrm{Na}^{+}, 3.1 \mathrm{mmol} / 1 \mathrm{~K}+$, $1.8 \mathrm{mmol} / 1 \mathrm{Ca}++, 216.9 \mathrm{mmol} / 1 \mathrm{Cl}^{-}, 0.2 \mathrm{mmol}^{2} / \mathrm{H}_{2} \mathrm{PO}_{4}^{-}$, 及 $1.8 \mathrm{mmol} / 1 \mathrm{HPO}_{4}^{-}$.

第 16 期

科学通

报

1265 


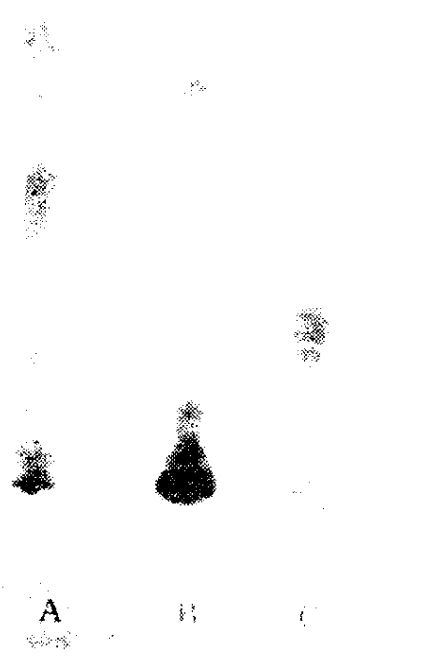

图 1 显示酪胺与神经节草脂结合的层析 谱及自显影

A. 猪脑神经节苦脂的海层层析徣，显示各个神 经节葉脂；B. 自显影,显示 GD3 及 GT1b 均 巳获得 [ $\left.{ }^{3} \mathrm{H}\right]$ 酪胺的故射性标志; C. $\left[{ }^{3} \mathrm{H}\right]$ 酪 肢的放射自显影

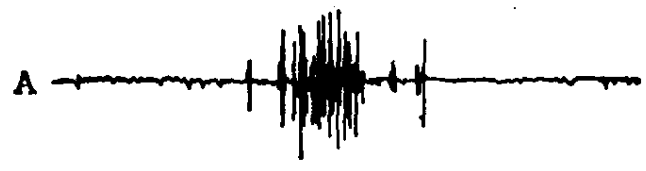

B

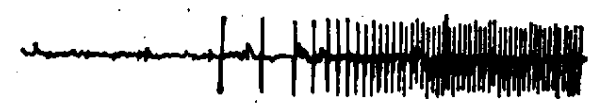

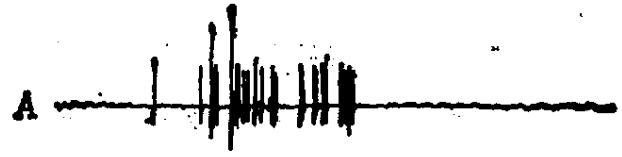

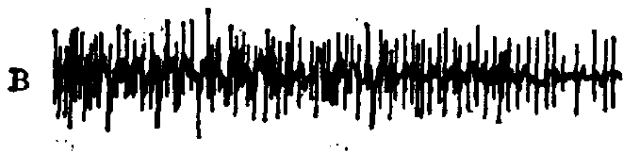

c

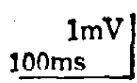

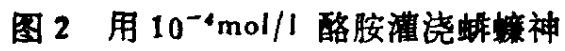
经的神经反应
A. $0 \mathrm{~min}$
B. $21 \mathrm{~min}$
c. $23 \mathrm{~min}$

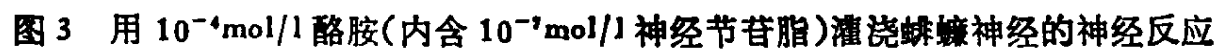
A. Omin;
B. 50 min;
C. $57 \mathrm{~min}$;
D. 洗去签绕洨

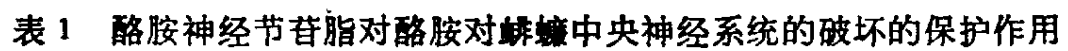

\begin{tabular}{c|c}
\hline 处理 $(\mathrm{mol} / \mathrm{l})$ & 阻断时间 (min) (四次试检平均结果) \\
\hline 酪胺 $10^{-4}$ & $23.8 \pm 2.8$ \\
酪胺 $10^{-4}$ & $57.6 \pm 3.8$ \\
内含神经节苦简 $10^{-7}$ & \\
\hline
\end{tabular}

t㭘验， $p<0.01$, 极显著.

\section{三、讨 论}

自从 Woolley 及 Gommi ${ }^{[1]}{ }^{[1}$ 早的发现, 用神经氨酸酶处理的真菌制备对 5-羟色胺的敏感 生可以用加人神经节菖脂(主要为 GD3) 来饭复, 这个问题, 即神经节苷脂是否是 5-羟色胺的 
受体迄今没有肯定 ${ }^{[n]}$. Ochoa 及 Bangham 相信 ${ }^{[8]}$, 神经节葉脂中的唾液酸就是这受体的作胢部 位,因为其他含有唾液酸的糖共轮物 (如 fetuin) 也能与 5-羟色胺结合.这些作者能够显示出, 5-羟色胺可以被含有各种神经节苦脂的脂质体所固定 (其亲和力为 $10^{2} \mathrm{l} / \mathrm{mol}$ ), '而不影响到膜 的离子通诱性. 另一方面, Maggio 等报道 ${ }^{[9]}, 5$-埥色胺及其他生物胺可以诱导含有神经节苷 脂的脂质体释放出葡萄糖来. Dette 及 Weseman ${ }^{[10]}$ 也证明了, 5-埥色胺的运转系统涉及到结合 的唾液酸, 用神经氨酸酶预先处理, 可以非竞争性地抑制 5-羟色胺被大鼠脑的突触体的高度 吸取,上文也已指出, 核磁共振的研究也显示出, 在 5-羟色胺与神经节苷脂之间有一个强烈的 交互作用,即在 $60 \mathrm{MHz}$ 处胺的芳族共振有一个显著的加宽 ${ }^{[2]}$. 氯丙嗪具有抗 5-䍩色胺的效 应，但也与神经节苷脂结合 ${ }^{[2]}$.

虽然神经节苦脂是否是 5-羟色胺的受体这一问题还没有解决，但是无疑神经节苷脂与 5羟色胺(也许与其他生物胺)有某种相互作用. 我们这一试验结果显示出, 猪脑神经节苷脂(特 别是 GD3 与 GT1b) 与酪胺(另一个生物胺)之间有相互作用; 并且初步用 $\left[{ }^{3} \mathrm{H}\right]$ 章鱼胺的 试验也证明了有同样情况，章鱼胺是又一个生物胺的神经递质及神经激素(末发表结果). 虽 然, 我们的试验结果不能肯定地证明神经节苷脂是这些生物胺的受体,但却证明了神经节菖脂 可以与生物胺结合.

McIlwain 早期预言 ${ }^{[11]}$ ，神经节苷脂对鱼精蛋白的保护作用乃是由于它能与鱼精蛋白结 合，形成一个复合物。这对于神经节苷脂也能保护这些有神经活性的生物胺是一个引人入胜 的假说. 我们的试验结果, 显示出在单向薄层层析谱上有两个新带出现, 它们极可能是 $\left[{ }^{3} \mathrm{H}\right]-$ 酪胺与 GD3 及 GT1b 结合的复合物. 双向层析后的液体闪婑的结果也同样, 这可能也证明 了神经节苷脂与生物胺之间有某些相互作用。

电生理的研究证明了神经节苦脂对酪胺、对神经阻断有一定保护作用,这极可能就是由于 神经节苷脂把酪胺结合了,而结合形成的复合物失去了活性.

酪胺对于脊椎动物的毒性极低，但对于昆虫却是一个有效的神经毒素. 我们已在昆虫中 发现了神经节苷脂的存在 (高平、张宗炳, 待发表结果), 但其含量很少, 仅为脊椎动物脑中的三 百分之一到一千分之一。既然, 神经节苷脂对酪胺的破坏神经传导有一定保护作用,这可能说 明了昆虫对酪胺的高度敏感性, 而脊椎动物有更大的耐受力.

[1] Wooley, D. W. \& Gommi, B. W., Nature, 202(1964), 1074; Proc. Natl. Acad. Sci., 53(1965), 957-963.

[2] Krishna, K. B. \& Balaram, D., FEBS Letters, 63(1976), 313-315.

[3] Mcllwain, H., Chemical Exploration of the Brain, Elsevier, Amsterdam, 1960.

[ 4] Chang, J. T. et al., Acta Entomol. Sinica, 27(1984), 15-23.

[5] Ledeen, R. W. \& Yu, R. K., Methods in Enzymolo gy, 83(1982), 139-191.

[6] Svennerholm, L., Biochem. Biophys. Acta, 617(1957), 604-611.

[7] van Heyningen, W. E., Nature, 249(1974), 415-417.

[8] Ochoa, E. K. \& Bangham, A. J., Neurochem., 26(1976), 1193-1198.

[ 9 ] Maggio, B. et al., Biochem. Biophys. Res. Comm, 7 7(1977), 1265-1277.

[10] Dette, G. A. \& Waseman, W., Hoppe Seylers Z. Physiol. Chem, 359(1978), 399-405.

[11] Mcllwain, H., Biochem. J. 78(1971), 24. 